This item was submitted to Loughborough's Research Repository by the author.

Items in Figshare are protected by copyright, with all rights reserved, unless otherwise indicated.

\title{
A systematic analysis of excitonic properties to seek optimal singlet fission: the BN-substitution patterns in tetracene
}

PLEASE CITE THE PUBLISHED VERSION

https://doi.org/10.1039/c9tc06581d

\section{PUBLISHER}

Royal Society of Chemistry (RSC)

VERSION

AM (Accepted Manuscript)

\section{PUBLISHER STATEMENT}

This paper was accepted for publication in the journal Journal of Materials Chemistry $\mathrm{C}$ and the definitive published version is available at https://doi.org/10.1039/c9tc06581d

LICENCE

CC BY-NC-ND 4.0

\section{REPOSITORY RECORD}

Pinheiro, Max, Francisco BC Machado, Felix Plasser, Adelia Aquino, and Hans Lischka. 2020. "A Systematic Analysis of Excitonic Properties to Seek Optimal Singlet Fission: The Bn-substitution Patterns in Tetracene”. Loughborough University. https://hdl.handle.net/2134/12191712.v1. 


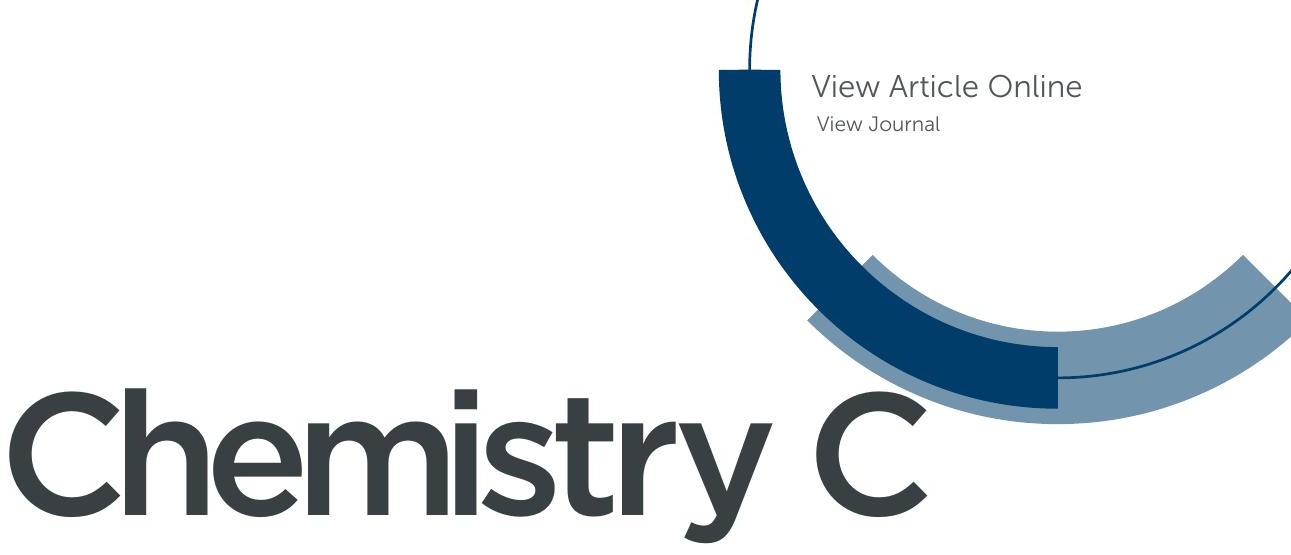

Materials for optical, magnetic and electronic devices

\section{Accepted Manuscript}

This article can be cited before page numbers have been issued, to do this please use: M. Pinheiro Jr, F.

B.C. Machado, F. Plasser, A. Aquino and H. Lischka, J. Mater. Chem. C, 2020, DOI: 10.1039/C9TC06581D.
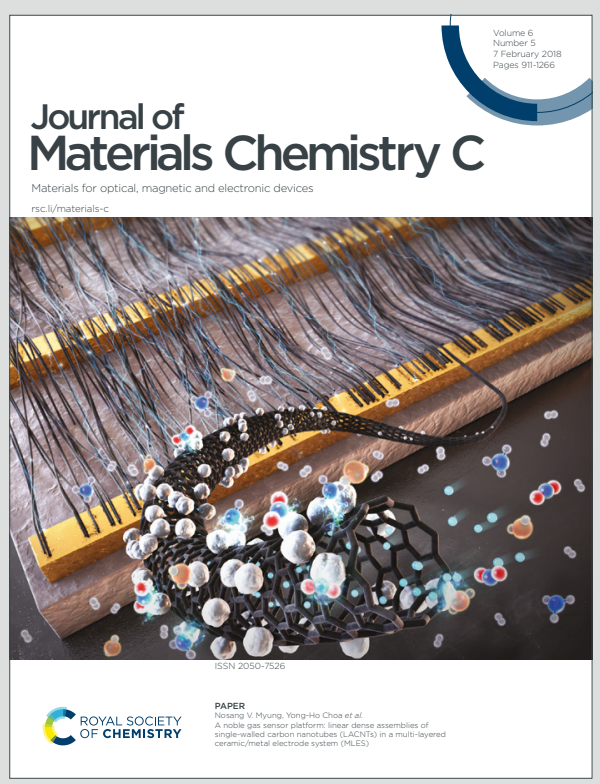

This is an Accepted Manuscript, which has been through the Royal Society of Chemistry peer review process and has been accepted for publication.

Accepted Manuscripts are published online shortly after acceptance, before technical editing, formatting and proof reading. Using this free service, authors can make their results available to the community, in citable form, before we publish the edited article. We will replace this Accepted Manuscript with the edited and formatted Advance Article as soon as it is available.

You can find more information about Accepted Manuscripts in the Information for Authors.

Please note that technical editing may introduce minor changes to the text and/or graphics, which may alter content. The journal's standard Terms \& Conditions and the Ethical guidelines still apply. In no event shall the Royal Society of Chemistry be held responsible for any errors or omissions in this Accepted Manuscript or any consequences arising from the use of any information it contains. 


\title{
Journal Name
}

\section{ARTICLE TYPE}

\section{A systematic analysis of excitonic properties to seek optimal singlet fission: the BN-substitution patterns in tetracene ${ }^{\dagger}$}

\author{
Max Pinheiro Jr, ${ }^{* a}$ Francisco B. C. Machado, ${ }^{* a}$, Felix Plasser, ${ }^{b}$ Adélia J. A. Aquino ${ }^{\text {cde }}$ \\ and Hans Lischka*cde
}

Received Date

Accepted Date

DOI: $00.0000 / x x x x x x x x x x$

\begin{abstract}
The development of efficient organic-based photovoltaic devices is a vibrant area of research with the potential of providing a cheap source of sustainable energy to society. The attainable power conversion efficiencies could be strongly enhanced via the singlet fission (SF) mechanism, a quantum mechanical phenomenon that potentially doubles the number of electron-hole pairs in a photoexcitation process by splitting a high energy singlet into two triplets. Biradicaloid molecules are particularly appealing for SF applications due to the possibility of controlling the balance between open-shell and closed-shell resonance structures via chemical modifications, which open new opportunities to fine tune the singlet and triplet excitation energies, and thus maximize the SF efficiency. Recently, we have shown that doping acenes with boron (B) or nitrogen (N) atoms leads to a large modulation in its biradicaloid nature at the ground-state. Herein, this previous study is extended to the case of asymmetric substitutions by introducing a BN-pair in a tetracene molecule to form azaborine analogues of acenes. The consequences of the chemical doping on the excitonic properties of tetracene is investigated through high-level multireference calculations. From a pool of 60 proposed BN-tetracene chromophores, we identify 15 new promising candidates for SF as they satisfy the energy level matching conditions involving the low-lying singlet and triplet states of a monomer. Still, some of these compounds show good chemical stability as evidenced by their modest biradical character. These results are interpreted in terms of aromaticity changes, charge transfer effects and exciton properties. More generally, this study shows how the energetics of singlet fission materials can be dramatically altered by using fairly simple chemical substitutions and provides detailed insight into the underlying relationships between the molecular structure, the electronic structure, and the excited state energies.
\end{abstract}

\section{Introduction}

The intricate quantum nature of materials at the nanoscale has proven to be a plentiful source of astonishing phenomena that has inspired the development of highly advanced technologies. Among these phenomena, singlet fission (SF) 1 1.4, a multichromophoric photophysical process whereby a singlet $\left(\mathrm{S}_{1}\right)$ exciton is spontaneously converted into two low-energy triplets, is a fascinating example which offers bright prospects for solar energy exploitation. While the most efficient silicon-based single junction solar cell is constrained by the Shockley-Queisser limit to power conversion efficiencies below $33 \%$, this value can be raised to $46 \%$ via SF due to the ability of doubling the number of low energy excitons per absorbed photon -12 . Importantly, triplet excitons are featured by a longer lifetime compared to singlet ones due to the spin selection rule. As a consequence, long exciton diffusion lengths are expected to occur, thereby facilitating the exciton's migration towards the donor/acceptor interface, finally enhancing the density of charge carriers collected at the electrodes 13 . Furthermore, the fact that SF is mostly linked to organic materials leads to especially appealing features such as low weight, good processability and mechanical flexibility that make the organic-based solar cells even more attractive for practical applications. Despite the notable progress in SF research, the limited availability of compounds combining an efficient SF rate with adequate chemical stability has hampered the development of SFbased light harvesting materials for real photovoltaic devices.

From a thermodynamic viewpoint SF chromophores need to fulfill two energetic criteria ${ }^{1}$ : (i) the first triplet state must lie between the ground $\left(\mathrm{S}_{0}\right)$ and lowest spin-singlet $\left(\mathrm{S}_{1}\right)$ excited state, ideally with half of the $S_{1}$ energy such that the energy released by one chromophore during the $S_{1} T_{1}$ decay is completely absorbed by one of the neighboring ground-state molecules, which is then 
excited to $\mathrm{T}_{1}$. Within this resonant condition, given by

$$
E\left(S_{1}\right)=2 E\left(T_{1}\right),
$$

$\mathrm{SF}$ is expected to proceed with maximum yield due to the isoergicity, whereas a highly exoergic situation is known to degrade the power conversion efficiency of SF-based solar cells since the excess energy is mostly dissipated as heat. (ii) In order to prevent recombination of the triplet exciton pairs being converted to a singlet and a higher triplet, it is also required that the energy of the second triplet state be equal or greater than $2 \mathrm{E}\left(\mathrm{T}_{1}\right)$, i.e.,

$$
2 E\left(T_{1}\right) \leq E\left(T_{2}\right) .
$$

Taken together, conditions (i) and (ii) establish important guiding principles for the design of efficient SF materials.

In recent years, much of the effort within the singlet fission research area has been devoted to uncover novel molecules that present an ideal match of the aforementioned energetic requirements. Since the first observation of SF in crystalline anthracene ${ }^{14}$, the acenes family $12 \mid 15,17$ and some derivatives 18 19 have become the standard reference systems for singlet fission, as the evolution of low-lying excitation energies with increasing chain length tends to favor exoergic conditions. In fact, a transition from a slightly endothermic situation in crystalline tetracene ${ }^{20}$ with a small activation energy of about $60 \mathrm{meV}$, to an optimal exothermicity in pentacene $\frac{15 \mid 21}{12}$ has been observed experimentally. Other prominent examples of molecules capable to undergo SF include perylenediimide (PDI) 2223, 1,3-diphenylisobenzofuran 24 , carotenoids 25 , bithiophene ${ }^{26}$ and zethrenes 27 . Most of these SF-compounds share a common feature, that is the diradical character, i.e., they carry unpaired electron densities to some extent. Indeed, it has been shown that the relationship between the singlet and triplet excitation energies is intimately connected with the diradical character 2829 , which is a quantum chemical descriptor of the relative contribution of openshell resonance configurations to the overall ground-state. While diradical character is not an observable quantity, its relationship with singlet-triplet (ST) energy gaps clearly provides a great advantage from a theoretical perspective in avoiding the cumbersome and expensive task of developing new SF chromophores by purely synthetic means. Finally, we note that excited-state aromaticity has been suggested ${ }^{30}$ but also disputed ${ }^{31}$ as an alternative design strategy for efficient SF materials.

Owing to its merit, the idea of tuning multiradical character has been well explored in the theoretical literature (see references 32 and ${ }^{33}$ for a recent review) as an effective strategy to balance the excitation energies of chromophores into desirable proportions to enable SF. In general, the larger the diradical character of a given chromophore, the smaller its ST energy gap is expected to be, whereas tetraradical character is more likely to affect higher singlet states. Accordingly, molecules that combine a weak to moderate diradical character with a fairly small tetraradical character are expected to satisfy the energy level matching conditions (i) and (ii) 29. This was shown to be the case for a diversity of molecular systems through computational studies, which explored different strategies, such as chemical modifications with aromatic-ring attachment and $\pi$-plane twisting 34 , heteroatoms substitution $35 / 36$ and annelation of antiaromatic carbon-rings 37 , to disturb the diradical character towards maximal SF rate. In particular, a perturbation strategy based on the captodative effect of BN substitution has been proposed as a pioneering theoretical effort $\frac{38}{38}$ in designing small $\pi$-conjugated molecules that combines the desired diradical character and kinetic persistence for SF. We have also contributed to this idea by using high-level quantum chemical computations to demonstrate that a wide scale of open-shell character can be attainable by doping oligoacenes with boron or nitrogen atoms. $\frac{39}{39}$ While such study was centered on the chemical stability of the ground-state systems, an important step further is to investigate how B/N substitutions in acenes may affect the distribution of energy levels relevant for SF.

Aside from the energetic perspective, a comprehensive characterization of the excited states in the first stage of singlet fission is also important as it provides an insightful view on the nature of electronic transitions and charge rearrangement in the excitation process. For instance, the average electron-hole separation gives a measure of the spatial distribution of the bound electronhole pair in the singly excited state which is a key property to distinguish between charge transfer (CT) or localized excitonic states in molecular systems 40 . In general, largely delocalized excitons are expected to have smaller binding energies, thus, facilitating the dissociation of the electrons and holes into free carriers, a prerequisite for efficient photovoltaic devices. Such excitonic effects can be quantitatively accessed in a molecular-orbital picture through quantum chemical excited-state calculations by using the one-particle transition density matrix as an approximation to represent the exciton wavefunction $\underline{4143}$. In addition, other analyses based on difference density matrices can reveal details about orbital relaxation effects of the molecular system upon excitation. $44 \mid 45$

Herein, we report a systematic and comprehensive $a b$ initio investigation on the electronic excited state properties linked to singlet fission for a series of BN-substituted tetracene molecules. In light of the complexity that naturally arises from the openshell character of these polyaromatic systems with a considerable amount of unpaired electrons, careful consideration on the methodologies adopted in the theoretical treatment has to be given to obtain reliable results. Accordingly, we adopt two stateof-art quantum chemical methods, NEVPT2 and MR-CISD, which are known to provide an accurate description of strong electron correlation inherent to radicaloid systems within a many-body picture, and still allow to treat ground and excited states on equal footing. Our main aim is to explore the energetic relation of singlet and triplet states as tools to scan a manifold of doped tetracene structures using the vertical excitation energies as descriptors. While SF is intrinsically an adiabatic process, the geometry optimizations necessary in the excited states to obtain adiabatic energies are computationally unaffordable for the level of theory and large database of medium-sized acene molecules considered in this work. Therefore, for the purpose of analyzing trends and obtaining mechanistic insight, we use the simple vertical approximation to compute the excitation energies related to the SF conditions (i) and (ii) for a set of $60 \mathrm{BN}$-doped tetracenes. 
These results are then used to perform a pre-screening for promising SF chromophores. The relationship between the extent of biradical character, given by the number of effectively unpaired electrons, and excitation energies is discussed. This survey allows us to envisage a design strategy for applying the B,N doping in polyaromatic systems in order to obtain a good balance between chemical stability and SF efficiency. In addition, the present study provides quantum chemical insights into how the different BN-substitutions may affect the excitonic properties of tetracene. Based on these results, we discuss the possibility of exploiting exciton analysis as an additional criterion to design and select efficient SF molecules. It is certainly not possible to perform a purely in-silico design of a singlet fission absorber even with current advanced quantum chemical technology, but we hope that this work will stimulate future research by showing how fairly simple substitutions can have a dramatic effect on the energetics and by illuminating the interplay between the molecular structure, the electronic structure, and the excited-state energies.

\section{Computational details}

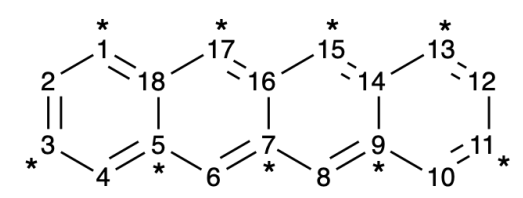

Fig. 1 Chemical structure of the tetracene molecule showing the numbering scheme used to generate and identify the doping configurations. The Coulson starred positions, corresponding to the odd numbers, are indicated by $\left({ }^{*}\right)$.

The set of chemically doped tetracene structures was generated by replacing two of the carbon atoms with one boron (B) and one nitrogen $(\mathrm{N})$ atoms. Following the numbering scheme shown in Fig. 1, we start with a B dopant fixed at position 1 while the $\mathrm{N}$ atom is moved from the site 2 until reaching the last doping site 18. This procedure is repeated by moving the B atom to the remaining doping sites, but in each step we remove all doping configurations which are equivalent by symmetry. It is worth noting that a simple permutation of the $\mathrm{B}$ and $\mathrm{N}$ doping positions does not necessarily lead to equivalent structures. The final set of selected BN-tetracene molecules comprises 60 different doping configurations out of the 77 theoretically possible configurations. 46 Their chemical structure is represented in Figure S1 of the Supporting Information (SI).

All model geometries were fully relaxed at the second-order Møller-Plesset perturbation theory 47 level by optimizing the atomic coordinates in the singlet closed-shell state. The resolution of the identity approximation (RI-MP2) ${ }^{48}$ was employed in combination with the Ahlrichs's triple- $\zeta$ valence (TZV) quality basis set augmented by polarization functions $[2 d, 1 f]$, def2TZVP 49 . For tetracene and all BN-doped systems, the converged MP2 equilibrium geometries were found to be planar. Because of this, the $C_{S}$ symmetry was used to perform the next computations. The reliability of the MP2 geometry optimization has been investigated in our previous study 39 for boron and nitrogen doping in different acene oligomers, in which it was found that the RI-MP2 method produces accurate structural properties even in the case of highly open-shell systems.

Excited state properties of the relaxed tetracene molecule and its BN-doped derivatives were accessed by using two variants of the multireference approach, which differ with respect to the treatment of dynamical correlation. In both cases, complete active space self-consistent field, $\mathrm{CASSCF}^{50}$ calculations were performed first to construct the spin-symmetrized reference wave function that properly includes the static correlation inherent to singlet open-shell systems. The resulting molecular orbitals and configuration state functions (CSFs) were then used in the subsequent MR calculations, carried out with the second-order $n$ electron valence state perturbation theory (NEVPT2) $)^{\frac{51}{1}}$ and configuration interaction with singles and doubles excitations (MRCISD $\sqrt[52]{52}$ methods.

In the case of NEVPT2 calculations, a strongly contracted (SC) scheme was employed. The active space of the prior CASSCF procedure comprised twelve electrons correlated within twelve $\pi$-orbitals, CAS $(12,12)$. MP2 natural orbitals (NOs) ranked by their occupation numbers were used as input to construct the initial guess for the CASSCF calculations. Thus, the initial active space is composed by the six highest occupied NOs with occupations ranging from $1.92 e$ to $1.86 e$ and the six lowest unoccupied NOs having $0.12 e$ to $0.04 e$. Only the eighteen $1 s^{2}$ core orbitals (one per heavy element) were kept frozen in the CASSCF orbital optimization routine. For the excited state calculations, a stateaverage CASSCF scheme was adopted by considering five states of different spin multiplicities, namely three lowest singlets and two triplets, all these belonging to $\pi-\pi^{*}$ excitations, with equal weights. Additionally, vertical ionization potential and electron affinity were computed at the SC-NEVPT2 level for all structures by taking the total energy difference between the neutral and charged $( \pm 1 e)$ systems evaluated from independent calculations at the relaxed geometry of the neutral system. All NEVPT2 calculations were carried out using the def2-TZVP basis set within the RI approximation.

MR-CISD computations were performed with the aim of evaluating exciton properties, since this method allows to relax the electron density for both ground and excited states. In this case, due to the higher computational cost of the MR-CISD method, the active space size used for CASSCF and as MRCI reference space was reduced to eight electrons in eight $\pi$-orbitals, $\operatorname{CAS}(8,8)$. The initial active orbitals were taken from a previous Hartree-Fock calculation following the energetic ordering within the $\pi$-symmetry. All molecular orbitals were fully optimized at the CASSCF level without any freezing scheme. At the MR-CISD level, only the $\pi$ orbitals and a few $\sigma$-orbitals (six reference doubly occupied and six virtual orbitals) were considered. The remaining $\sigma$-orbitals were all kept frozen. The reference configurations at MRCI level were constructed using the mentioned $\operatorname{CAS}(8,8)$ in the $\pi$ space and included the six doubly occupied $\sigma$-orbitals. All single- and double-electron excitations generated by applying the interacting space restriction ${ }^{53}$ were then considered to create the MR-CISD expansion space. Calculations for different spin symmetries were carried out separately by considering three roots of the singlet ${ }^{1} \mathrm{~A}$ ' and two of the triplet ${ }^{3} \mathrm{~A}^{\prime}$ in a state-average fashion with equal 
weights. For comparison purposes, excitation energies were computed also at the MR-CISD level, and in this case, size-extensivity contributions were included by means of the renormalized Davidson correction $54[55$, denoted as + Q. The Pople's 6-31G(d) basis set was used throughout the CAS $(8,8) /$ MR-CISD calculations.

A series of quantum chemical descriptors were derived from the MR-CISD electron densities for both ground and excited states to elucidate the effects of BN-doping on the radical character and exciton properties. These are described in the following subsection.

The RI-MP2 and NEVPT2 calculations were performed employing the ORCA $4.0^{56}$ program package. For the MR-CISD calculations, the COLUMBUS program $\sqrt{57 / 59}$ was used in its parallel implementation 60 . Post-processing of the MR results was carried out using the TheoDORE program ${ }^{42 \mid 61}$ to evaluate the effective number of unpaired electrons and the exciton properties derived from the 1TDM.

\subsection{Wavefunction Analysis Methods}

Understanding and analyzing the wavefunctions produced in multireference calculations can be quite challenging especially when an extended set of different molecules is considered as is the case here. Some of us have developed a number of analysis strategies to automatise this task $\sqrt{40,42 / 62}$ and we shall only give a very brief overview of the methods used, here.

To analyze the biradical character, the main concern in this work, we have used the unpaired density and the total number of effectively unpaired electrons, $\mathrm{N}_{U}$, following Head-Gordon's approach. ${ }^{63}$ Specifically, we use the non-linear expression

$$
N_{U}=\sum_{i=1}^{M} n_{i}^{2}\left(2-n_{i}\right)^{2},
$$

with $n_{i}$ being the occupation of the $i^{t h}$ natural orbital (NO) of the state of interest and the summation runs over all $M$ NOs. This nonlinear model allows to emphasize the contributions from orbitals with occupation near one and damps contributions that are nearly doubly occupied or nearly unoccupied. In the same spirit we compute a density of unpaired electrons as

$$
\rho_{U}(r)=\sum_{i=1}^{M} n_{i}^{2}\left(2-n_{i}\right)^{2}\left|\psi_{i}(r)\right|^{2}
$$

where $\psi_{i}$ is the $i^{\text {th }}$ NO and $r$ refers to the coordinates in space.

To characterize the charge transfer occurring during the excitation process, we use an average electron-hole separation as initially defined in ref 40, and to which we refer as exciton size

$$
d_{e x c}=\sqrt{\frac{1}{\Omega} \sum_{A, B} \Omega_{A B} d_{A B}^{2}} .
$$

Here, the indices $A$ and $B$ run over the different pairs of atoms and their separation is given by $d_{A B} . \Omega_{A B}$ is the charge transfer number, ${ }^{43 / 64}$ representing the weight of the $A \rightarrow B$ transition in the overall excitation process, and $\Omega$ is a normalisation factor, which indicates the weight of single excitations in the overall transition process. 42
Finally, the rearrangement of the electron density during the excitation process is represented by the attachment-detachment analysis proposed by Head-Gordon et al ${ }^{65}$. Briefly spoken, this analysis proceeds by a diagonalization of the difference density matrix between the two states of interest. All contributions corresponding to negative eigenvalues are subsequently grouped together to form the detachment density, representing the excitation hole, and the contributions with positive eigenvalues form the attachment density (excited electron) ${ }^{42}$ For neutral excitations, i.e., when the total number of electrons is conserved in a transition, it holds that the spatial integral over the detachment and attachment densities is the same and we denote this integral as the promotion number $p$. In the case of configuration interaction singles, $p$ is always exactly to 1 , but for correlated wavefunction models one often finds that $p$ is significantly larger than 1 , a feature that can be associated with double-excitation character $\sqrt{66}$ and orbital relaxation effects $44 / 45 / 67$.

\section{Results and Discussion}

\subsection{Analysis of bonding patterns}

The versatility of the BN-substituted systems derives from the possibility of using two distinct design principles to tune their properties. On the one hand, we can choose whether the two atoms are taken out of the same or different sublattices of the underlying graphene structure. On the other hand, the properties depend on whether Clar sextets ${ }^{68}$ can be formed at all and whether their formation creates radical centers.
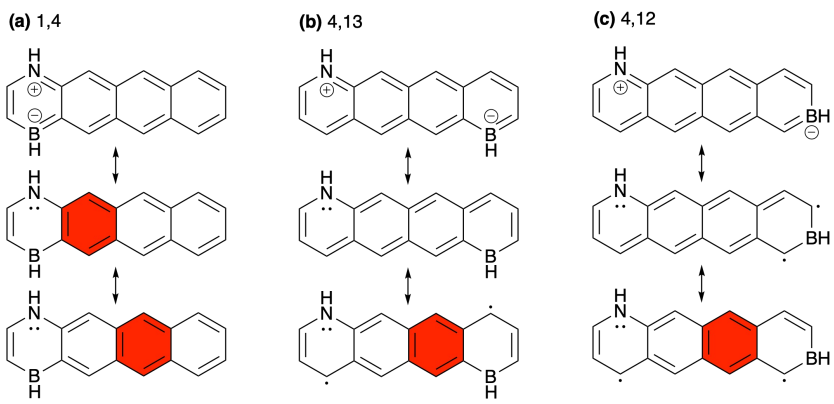

Fig. 2 Illustration of possible bonding patterns in BN-substituted tetracenes (Clar sextets shown in red): (a) neutral closed-shell resonance structure containing a Clar sextet available, (b) neutral closed-shell resonance structures possible but Clar sextet can only be formed by introduction of radical centers, (c) no neutral closed-shell resonance structure possible.

Tetracene, like all alternant hydrocarbons, can be viewed as being composed of two sublattices where chemical bonds occur only between but not within the sublattices. In Fig. 1 1 one of these sublattices is marked by Coulson's star notation and it is noted that all the starred positions correspond to odd numbers in our numbering scheme. Thus, any (odd, odd) or (even, even) substitution corresponds to the case where $\mathrm{B}$ and $\mathrm{N}$ are in the same sublattice and vice versa for (odd, even) and (even, odd) substitution. It is now important to realise that it is only possible to draw a resonance structure without formal charges or radical centers if the $\mathrm{B}$ and $\mathrm{N}$ atoms are placed into different sublattices, i.e. one is in an odd and the other one in an even position in our numbering 
scheme. This is illustrated in Fig. 2 for three representative subsitution patterns: 1,$4 ; 4,13 ; 18,12$. The first formal step in BN substitution may be performed by leaving the bonding pattern from Fig. 1 unchanged and adding the $\mathrm{B}$ and $\mathrm{N}$ atoms with formal negative and positive charges, respectively, as illustrated in the first row of Fig. 2. If the $\mathrm{B}$ and $\mathrm{N}$ atoms are on different sublattices, then it is possible to flip the double bonds to create a resonance structure where one of the double bonds is converted into a lone pair on nitrogen (Fig.2 (a,b) second row). If they are on the same sublattice, then it is not possible to do so without the formation of radical centers as shown in Fig. 2 (c). Cases (a) and (b) are distinguished by whether it is possible to draw a neutral closedshell resonance structure that also contains a Clar sextet. This is possible for 1,4 while the formation of a Clar sextet requires the formation of a pair of radical centers for 4,13. In line with our previous analysis of various unsubstituted polycyclic aromatic hydrocarbons ${ }^{69}$ we, thus, expect the following ordering in terms of diradical character for the three exemplary cases: (a) $<$ (b) $<$ (c). The strongest diradical character is obtained if it is not possible at all to draw a neutral resonance structure without radical centers while also the formation of Clar sextets may present itself as a crucial driving force for creating diradical character. The first case can be distinguished by whether the indices of the substituted atoms are both odd or both even while the second case requires a more detailed consideration of resonance structures.

\subsection{Diradical character and Excitation Energies}

The relation between the singlet and triplet excitation energies computed within the vertical approximation are shown in Figure 3 (see Table S1 for numerical values) together with the multiradical character represented by a color map. Noteworthy, the relatively modest diradicaloid character of pristine tetracene $\left(\mathrm{N}_{U}\right.$ $=0.37 e$, diamond marker in Fig. 3 is strongly modulated by varying the positions of the BN dopants. The largest open-shell character $\left(\mathrm{N}_{U}=1.26 \mathrm{e}\right)$ is obtained for the (even, even) doping pattern 4,10, which in line with Fig. 2 (c) does not possess a neutral resonance structure without radical centers. The smallest open-shell character $\left(\mathrm{N}_{U}=0.21 e\right)$ is obtained for the (even, odd) doping pattern 6,15, which conforms with Fig. 2 (a) possessing a neutral closed-shell resonance structure containing two Clar sextets. The diversity in open-shell character is reflected in the broad range of calculated singlet-triplet (ST) energy gaps, largely distributed between $0.7 \mathrm{eV}$ and $2.0 \mathrm{eV}$. Molecules having a larger number of unpaired electrons, i.e., larger radicaloid character at the ground-state are generally expected to show lower ST gaps. For BN-tetracenes, this is not always the case, as can be observed for the 6,8 doping configuration, for example, which has approximately half of the ST gap of that of the 1,3 structure, although this latter has a slightly larger fraction of unpaired electrons $\left(\mathrm{N}_{U}(6,8)=0.46 e\right.$ and $\left.\mathrm{N}_{U}(1,3)=0.54 e\right)$.

BN doping is also quite effective in modulating the first singlet excitation energy, $E_{S 1}$, of tetracene in such a way that it can push the $2 T_{1}-S_{1}$ energy difference towards exoergic or even isoergic situations. Experimental studies on solid tetracene have shown that $\mathrm{SF}$ occurs under endothermic conditions ${ }^{70}$, i.e., $2 \mathrm{E}_{T 1^{-}}$
$E_{S 1}>0$, a feature reproduced by our calculations (see Figure 3). The $S_{1}$ energy of tetracene obtained using NEVPT2 $\left(E_{S 1}=\right.$ $2.53 \mathrm{eV}$ ) is quite close to the experimental gas-phase reference, $2.88 \mathrm{eV} 71$, and also agrees well with previous CASPT2 computations ${ }^{45}$. The NEVPT2 value of $1.87 \mathrm{eV}$ obtained here shows good agreement with the vertical ST gap estimated using the CCSD(T)/CBS method $(1.76 \mathrm{eV})$ 72. We therefore expect a similar level of accuracy also for the BN-doped counterparts.

Direct comparison of computed excitation energies for the doped systems with experiment is difficult because of the lack of spectroscopic gas phase data. Also, respective solution data are quite rare. We calculated the first singlet excitation energy, $\mathrm{E}_{S 1}$, for two compounds, namely $2-\mathrm{nBu}-1,2-\mathrm{BN}$-tetracene ${ }^{73}$ and 5,6Boraazaro-benz [a] anthracene ${ }^{74}$, for which experimental data are available. Note that the former molecule corresponds to the 1,2BN-doped structure of our database, except for the presence of the $n$-butyl group bonded to the boron atom, which should not strongly affect the optical properties. For 2-nBu-1,2-BN-tetracene, the $E_{S 1}$ value obtained with NEVPT2 $3.19 \mathrm{eV}$ compares favorably to the first band maximum measured in the UV-Vis absorption spectrum, $2.90 \mathrm{eV}$. An even better agreement between our calculation and the experiment is found for the 5,6-Boraazarobenz[a]anthracene: $3.87 \mathrm{eV}$ (calc.) and $3.91 \mathrm{eV}$ (exp.). Thus, these results demonstrate the reliability of our method not only for the pristine system but also for the BN-doped derivatives.

Within the full set of $60 \mathrm{BN}$-doped structures investigated, we found that at least 4 different molecules satisfy the exoergicity conditions for SF according to NEVPT2 calculations, whereas other 11 doping configurations show small endoergicity with energy differences $2 T_{1}-S_{1}$ below $0.3 \mathrm{eV}$. In this set of promising SF chromophores (see star markers in Figure 3 and highlights in Table S1), the 1,3 BN-tetracene is the only one having the energy level of $\mathrm{T}_{2}$ state lower than $2 \mathrm{E}\left(\mathrm{T}_{1}\right)$ and, therefore, might be susceptible to the detrimental triplet-triplet annihilation. It is noteworthy that all structures predicted as potentially active SF materials by the NEVPT2 method, also fulfill the SF energy conditions using MRCI with Pople's correction scheme for size extensivity, denoted as $+Q$ (see Table S2). The average of $S_{1}\left(T_{1}\right)$ excitation energy over the $60 \mathrm{BN}$-tetracene structures is $1.86 \pm 0.75 \mathrm{eV}$ $(1.16 \pm 0.56 \mathrm{eV})$ according to MRCI results, while NEVPT2 gives $1.80 \pm 0.72 \mathrm{eV}(1.23 \pm 0.52 \mathrm{eV})$. This overall agreement reflects the approximate linear correlation between the excitation energies estimated with NEVPT2 and MRCI, as evidenced in Fig. 4. It is interesting to note that MRCI gives lower $S_{1}\left(T_{1}\right)$ energies than NEVPT2 for 25 (44) structures. A statistical evaluation of the differences between these two methods is provided by the mean $a b$ solute error (MAE) and root-mean squared error calculated from the excitation energy differences, $\mathrm{E}_{i}(\mathrm{MRCI})-\mathrm{E}_{i}$ (NEVPT2) with $i=\mathrm{S}_{1}, \mathrm{~T}_{1}$ and $\mathrm{T}_{2}$, for all structures (see table 1 ). Although one can see a good accordance between MRCI and NEVPT2 for the $\mathrm{T}_{1}$ state, the MAE and RMSE observed for the singlet $\mathrm{S}_{1}$ state are larger, in line with general considerations regarding difficulties in describing the so-called ${ }^{1} L_{a}$ ionic states in conjugated $\pi$ systems. $\frac{67775176}{T}$ This leads to significant discrepancies between the two methods for estimating the first singlet fission condition (SFC1). Owing to the larger differences between the first ex- 

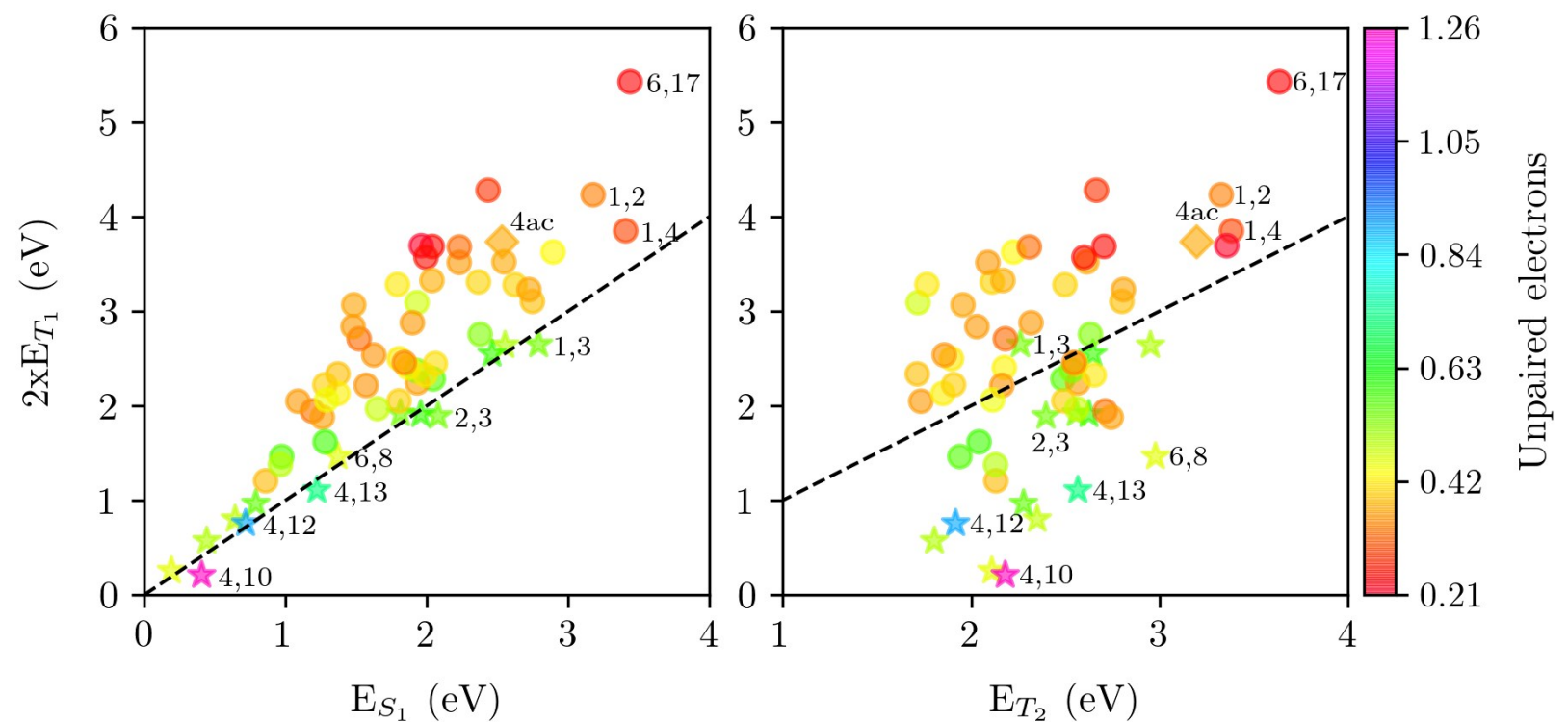

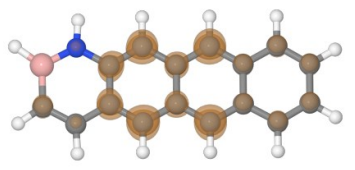

1,2

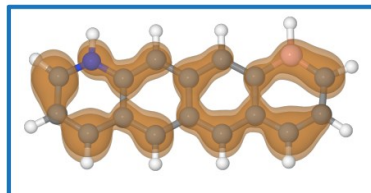

4,10

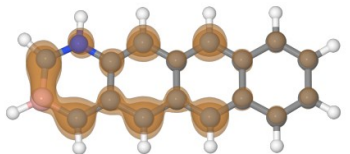

1,3

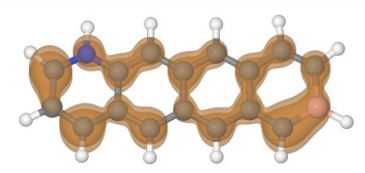

4,12

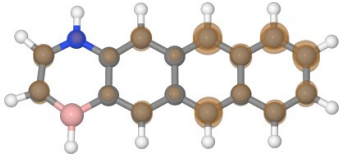

1,4

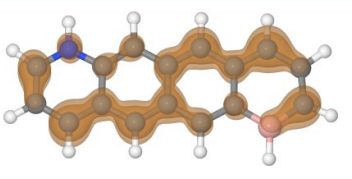

4,13

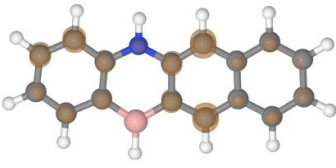

6,17

Fig. 3 Singlet fission energetics in BN-tetracenes. Top: Vertical singlet $\left(E_{S_{1}}\right)$ and triplet $\left(E_{T_{1}}\right.$ and $\left.E_{T_{2}}\right)$ excitation energies of the 60 BN-doped tetracene molecules calculated by using the CAS $(12,12) / N E V P T 2$ method. The dashed lines indicate the optimal energetic conditions $\left(2 \mathrm{E}_{T 1}=\mathrm{E}_{S 1}\right)$ for SF. The color map represents the total number of effectively unpaired electrons $\left(\mathrm{N}_{U}\right)$. Star markers are used to identify the optimal BN-tetracene candidates for SF. Bottom: Density of unpaired electrons for the lowest-energy singlet state of selected BN-doped systems.

cited singlet $\left(\mathrm{S}_{1}\right)$ and triplet $\left(\mathrm{T}_{1}\right)$ states obtained with MRCI, this method tends to favor an exoergic condition for SF as compared to NEVPT2. In fact, besides the 4 structures predicted by NEVPT2 as active SF chromophores, other 9 structures are also pointed out as potential SF candidates by our MRCI calculations, since they satisfy the required energetic conditions (see red highlights in Tables S1 and S2).

Table 1 Statistical analysis of the deviation between the excitation energies computed using the MR-CISD+Q (Pople's correction) and NEVPT2 methods for the whole series of BN-doped tetracene molecules.

\begin{tabular}{rcccc}
\hline & $\mathrm{S}_{1}$ & $\mathrm{~T}_{1}$ & $\mathrm{~T}_{2}$ & SFC1 \\
\hline MAE & 0.1828 & 0.1082 & 0.1236 & 0.2714 \\
RMSE & 0.2319 & 0.1461 & 0.1506 & 0.3147 \\
\hline
\end{tabular}

Importantly, a wide scale of diradical character is attainable (see Figure 3 and Figure S2 for unpaired density plots) within the subset of promising SF candidates, which opens up the possibility of devising highly efficient SF chromophores with low reactivity. For example, the 6,8 BN-tetracene molecule, which satisfies both SF conditions, presents a much smaller and localized unpaired electron density as compared to the also suitable 4,10, 4,12 and 4,13 chromophores (see blue rectangle in Fig. 3). Therefore, these three latter compounds are expected to exhibit higher chemical instability as also evidenced by their small ST energy gaps. Note that the three molecules 4,10, 4,12 and 6,8 with an (even, even) substitution pattern conform with with Fig. 2 (c) possessing no neutral closed-shell resonance structure whereas the large diradical character of 4,13 is explained in Fig. 2(b).

In addition to good chemical stability, it is also desirable for practical reasons that the energy of generated triplet excitons fall in the range of $\mathrm{E}_{T 1} \simeq 1.0-1.3 \mathrm{eV}$, with the singlet $\mathrm{E}_{S 1}$ twice larger to maximize the SF efficiency 1 177. This condition is accomplished by six different doping configurations: 2,3, 18,2, 18,4, 6,4, 18,3 and 3,18 (Table S1). Notably, although the 3,18 and 18,3 BNtetracene molecules exhibit approximately equal ST gaps, a simple interchange of $\mathrm{B}$ and $\mathrm{N}$ positions affects the $S_{1}$ state in such way that the energy level matching conditions change from endoergic $(0.11 \mathrm{eV})$ to slightly exoergic $(-0.05 \mathrm{eV})$. This influence the of BN-doping positions is even more striking in the case of 18,2 and 2,18 structures, where the BN permutation makes a 

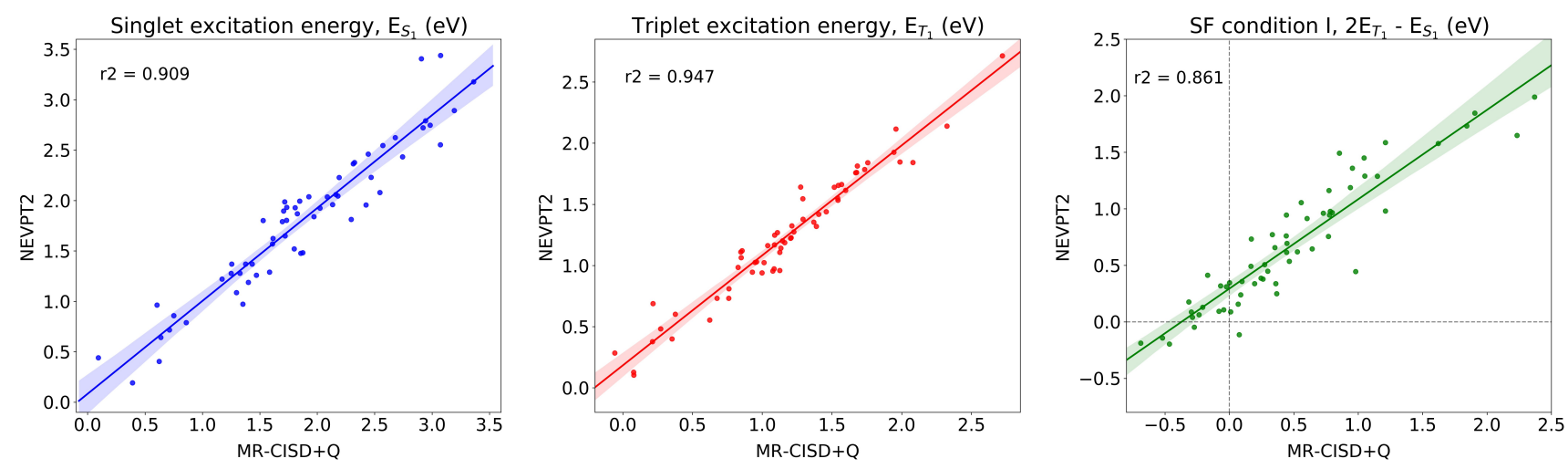

Fig. 4 Statistical correlation analysis. Scatter plots are used to display the relationship between the excitation energies estimated by the NEVPT2 and MR-CISD+Q methods. A fitted regression line with $98 \%$ confidence intervals (shaded regions) is shown demonstrating the linear correlation between the methods.

critical difference in the excitation energies, leading to a change from highly-active to inactive SF. It is also interesting that the 6,4 BN-tetracene, which exhibits the largest endoergicity within the pool of promising SF candidates, is the only compound having the two dopants in different rings. Based on these results, we conclude that the external rings are the most likely effective regions of tetracene to apply the BN-doping in order to tune the energy levels of excited states towards an ideal match of the SF conditions.

\subsection{Ionization Potential and Electron Affinity}

In the physical sense, ionization potential (IP) and electron affinity (EA) are key quantities intimately linked to the operation of organic devices, whereas in a chemical perspective they can be associated with electronic stability. The influence of BN-doping on the vertical IPs and EAs of tetracene is analyzed in Fig. 5 As one can see, there is a strong modulation in the one-particle removal/addition energies of the doped systems with varying the $\mathrm{B}$ and $\mathrm{N}$ positions along the tetracene backbone. For the pristine molecule, the IP and EA values computed with NEVPT2 method, $6.62 \mathrm{eV}$ and $1.10 \mathrm{eV}$, are in good agreement with gas-phase experimental data (6.97 ev ${ }^{78}$ and $1.04 \mathrm{eV}^{79}$ ). Most of the doping configurations (43 structures) contribute to destabilizing the systems as indicated by the smaller fundamental (IP-EA) gaps compared to pristine tetracene. This is especially evident for the doped structures with larger B,N separation, for example, in which the dopants occupy the opposite external rings of tetracene (from 4,10 to 3,12 in Fig. 5. In contrast, a significant stabilization effect is observed by doping tetracene at positions 5,14, 1,2 and 6,17, as indicated by the increase in its IP by $0.75 \mathrm{eV}, 0.65 \mathrm{eV}$ and $0.64 \mathrm{eV}$, respectively. This is in line with the weak diradical character observed for these three doped structures (see Figure S2).

Within the subset of 15 BN-tetracenes with optimal characteristic energy for singlet fission (see red and orange bars in Fig. 57, all doping configurations result in a reduction of the tetracene's fundamental gap by decreasing the IPs and increasing EAs; the only exception is the 18,4 structure which has a fundamental gap
$0.01 \mathrm{eV}$ larger than that of tetracene. This is a desirable feature for practical applications in SF solar cells design with multilayer structure, since smaller IPs contributes to reduce the energy offset at the interfaces with common acceptor materials, thereby facilitating the triplet dissociation. 480

\subsection{Excitonic effects}

Having identified the BN-substituted tetracene chromophores that satisfy the SF energy criteria, we turn our attention to investigating how excitonic properties of tetracene are modified upon chemical doping. In this respect, an important quantity is the exciton binding energy, as it represents the strength of the Coulomb interaction by which the electron and hole created upon excitation are bound. By definition, the binding energy of the lowestenergy exciton, $\mathrm{E}_{\text {bin }}$, can be calculated as the difference between the fundamental gap (i.e., $\mathrm{E}_{\text {gap }}=$ IP-EA) and the optical gap $81+83$. In the present case, we substitute the excitation energies of the $S_{1}$ and $\mathrm{T}_{1}$ states for the optical gap to compute the singlet and triplet exciton binding energies, respectively. Here, the triplet binding energies can be seen to represent the pure Coulomb attraction whereas for the singlets also a repulsive exchange term comes into play. 67

Figure 6 shows the $\mathrm{E}_{b i n}$ values obtained for the lowest singlet and triplet excited states using the NEVPT2 method for the subset of BN-tetracene molecules with optimal SF energetics (see also table S3). The singlet $\mathrm{E}_{\text {bin }}$ calculated for the isolated tetracene molecule is $2.99 \mathrm{eV}$ (dashed line in Fig. 6) whereas the triplet value is $3.65 \mathrm{eV}$ (dotted line in Fig. 6). Note that singlet compares favorably with the estimation derived from gas-phase experimental values for the IP, EA and optical gap: $\mathrm{E}_{b i n}=(6.97$ - 1.04) - 2.88 $=3.05 \mathrm{eV}$. By introducing the BN dopants at different positions, the singlet $\mathrm{E}_{b i n}$ can vary between $1.9 \mathrm{eV}$ and $4.3 \mathrm{eV}$ while the triplet values vary in the range of $2.4-4.7 \mathrm{eV}$ (table S3). As expected, the triplet $\mathrm{E}_{b i n}$ values appears systematically greater than the singlet ones due to the absence of exchange repulsion in the triplets ${ }^{84}$. Most of the doping configurations shown in Fig. 6 (10 structures) contribute to increasing the $\mathrm{E}_{b i n}$, resulting in tighter bound excitons compared to pristine tetracene. This feature is 


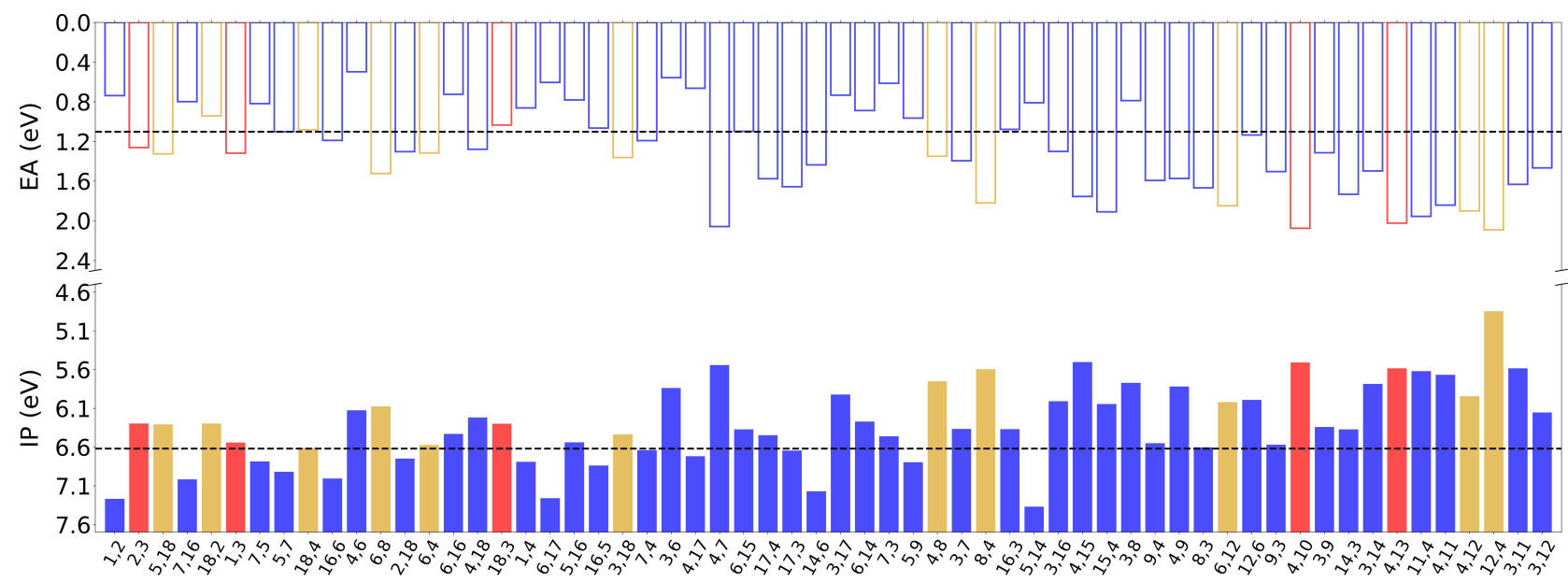

Fig. 5 Influence of BN-doping on the frontier energy levels. Ionization potential (IP) and electron affinity (EA) calculated for the whole set of BNdoped tetracenes using the CAS(12,12)/NEVPT2 approach (see also table S3). The horizontal dashed lines indicate the IP and EA values obtained for the pristine molecule. Red color are used to indicate the doped systems that exhibit exoergic SF, whereas those ones satisfying an endoergic SF condition below $0.3 \mathrm{eV}$ are highlighted in orange. The labels in the $\mathrm{x}$-axis are ordered according to the B, $\mathrm{N}$ separation from lowest to the largest.

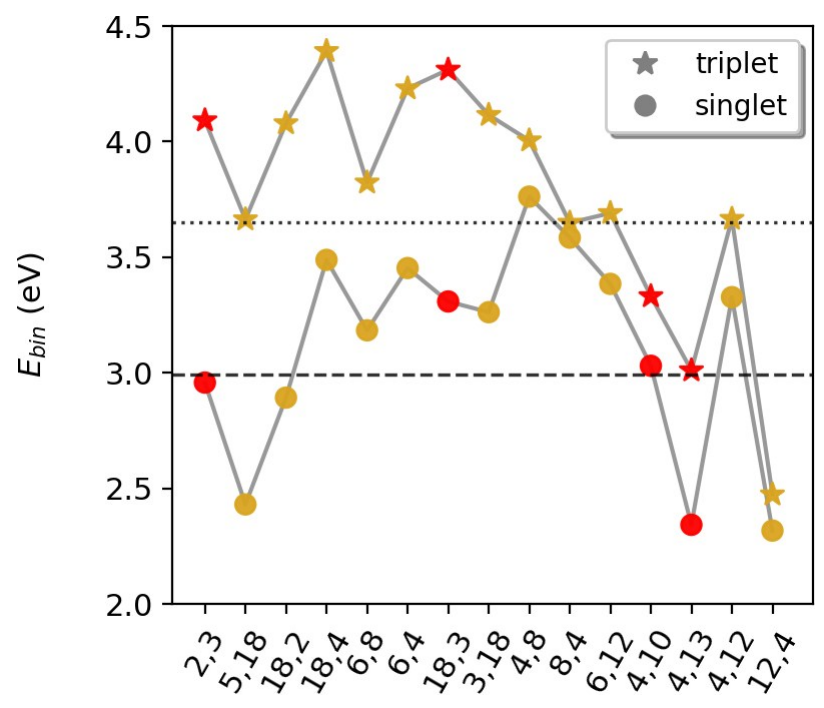

Fig. 6 Exciton energetics of BN-tetracenes. Singlet $(\bullet)$ and triplet $(\star)$ exciton binding energy of selected BN-doped tetracene molecules (see table S3 for the complete list) calculated as the difference between the fundamental gap and optical gap using the CAS(12,12)/NEVPT2 method. Red [orange] dots indicate molecules that satisfy an exoergic [slightly endoergic] condition for singlet fission.

detrimental for organic solar cell applications in general ${ }^{85}$, since higher energies are required for these systems to overcome their $\mathrm{E}_{\text {bin }}$, and thus dissociate the exciton into free charge carriers. On the other hand, the 5,18, 4,13 and 12,4 $\mathrm{BN}$ substitutions reduce the binding energy of the singlet excitons in tetracene by about $0.6 \mathrm{eV}$ and the 4,10, 4,13 and 12,4 substitutions notable reduce the triplet value.

Further insight into the nature of the electronically excited states in BN-tetracene compounds can be obtained by analyzing the exciton size, $\mathrm{d}_{\text {exc }}$. This quantity gives a measure of the dynamical charge separation in a molecular system derived from the interactions between different natural transition orbitals 62 . Accordingly, a large $\mathrm{d}_{\text {exc }}$ indicates weakly interacting electron-hole
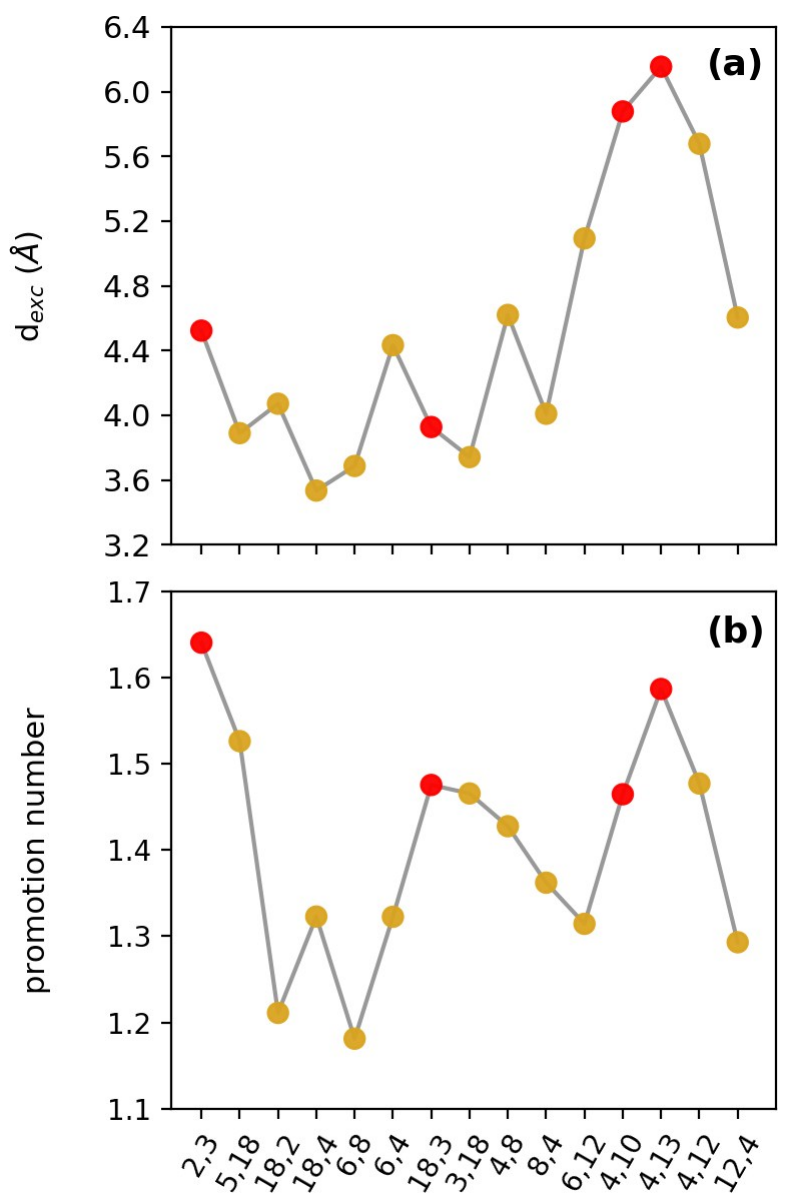

Fig. 7 Nature of the singlet exciton in BN-tetracenes. (a) Approximate exciton sizes $\left(\mathrm{d}_{e x c}, \AA\right)$ and (b) promotion numbers of the BN-doped tetracene molecules with optimal singlet fission energetics (see table S3 for the whole list of doped systems) computed at the CAS(8,8)/MR-CISD level of theory. Red [orange] dots indicate molecules that satisfy an exoergic [slightly endoergic] condition for singlet fission. 
pair. The $\mathrm{d}_{\text {exc }}$ values obtained from MR-CISD calculations using equation (3) are listed in table S3 for all BN-doped structures and displayed in Fig. 7p only for the systems matching the SF energy criteria. As a general trend, we found that the size of the singlet exciton oscillates between $3.5 \AA$ and $4.7 \AA$ for most of the BN-doping configurations (48 molecules, table S3). This indicates that the introduction of BN defects in tetracene favors the confinement of the electron-hole pair within a region that spans approximately the length of two fused hexagons. For the subset of optimal SF candidates shown in Fig. 79, only four structures $(6,12,4,10,4,12$ and 4,13) have a dynamic exciton size larger than $4.8 \AA$. The $4,13 \mathrm{BN}$-tetracene molecule has the most delocalized exciton, with an RMS size of $6.15 \AA$.

The variation of the promotion number $p$ with respect to the positions of the BN dopants is plotted in Fig. 77 (see table S3 for all BN-doped systems). This quantity is particularly helpful to identify other relevant effects beyond the simple picture of oneelectron transitions, which can be related to double-excitation character or orbital-relaxation effects ${ }^{42 / 45] 62}$. The $p$ values vary from 1.10 to 1.64 along the full series of BN-tetracene molecules. Interestingly, for the doped systems featuring exoergic singlet fission (red circles in Fig. 7p), the total number of electrons rearranged given by $p$ is somehow enhanced. Since for these molecules the $\Omega$ values are considerably small $(\sim 0.57$, see table S3), the double excitation character should play a more important role in the rearrangement of the excited electrons. By contrast, the most stable BN-tetracene molecules with respect to the number of unpaired electrons exhibit larger $\Omega$ values $(\Omega(6,15$ and 6,17$) \approx 0.76$ ), indicating a predominance of single excitation character.

Complementary to the above discussion, we perform an attachment/detachment density analysis to obtain a better understanding of the effects of BN-doping on the spatial distribution of the electron and hole densities. Inspecting the plots in Fig. 8, one can see that both electron and hole densities change from a fully delocalized (as in the 2,3 and 4,10 structures) to a more compact shape by varying the $\mathrm{BN}$ positions. These changes should be kept in mind when engineering SF materials with optimal intermolecular orbital overlap. These analyses also show that during the excitation process the B atom can either act as an acceptor or as a donor relative to the electron distribution in the ground state. For example, in the case of the 8,4 BN-tetracene, the detachment density, which describes removal of charge from the ground-state density, shows a large contribution by the boron orbitals, whereas they do not participate in the attachment density, which represents the added charge distribution to the rearranged density in the excited state. As a result, a net charge-transfer from boron to the carbons and nitrogen atoms is expected to occur upon excitation. This is to be interpreted in the sense that the overall acceptor character of the $B$ atom is reduced when going from $S_{0}$ to $S_{1}$. The opposite situation is found for the 4,10 doping configuration, in which the full contribution of boron orbitals occurring only in the attachment density indicates an acceptor character for this doping species. Moreover, because of the large overlap between the detachment and attachment densities observed in most of the BN-tetracene molecules, the $S_{0} \rightarrow S_{1}$ electronic excitation should

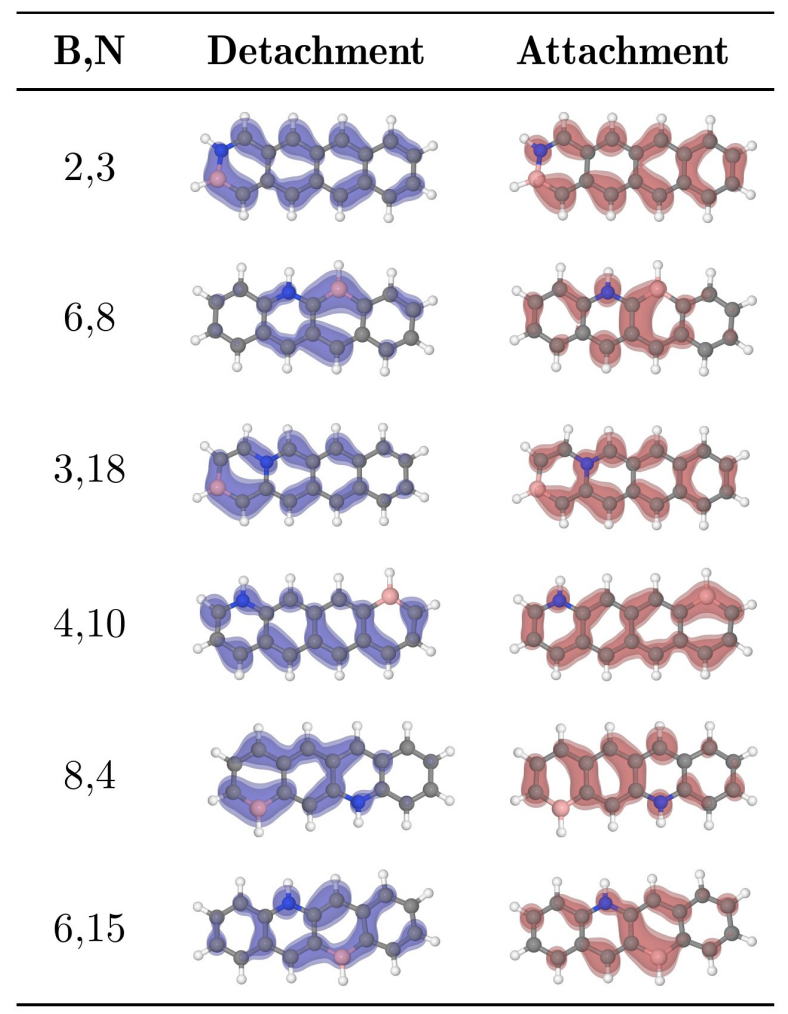

Fig. 8 Electron and hole distributions in BN-tetracenes. Attachment/detachment density plots of selected BN-doped tetracene compounds calculated for the low-lying singlet electronic transition, $S_{0} \rightarrow S_{1}$, at the CAS $(8,8) / M R-C I S D$ level of theory.

exhibit low charge-transfer character 86 .

\section{Conclusions}

The effectiveness of chemical doping in tetracene by nitrogen and boron atoms as a promising strategy for the pursuit of highly efficient singlet fission sensitizers has been clearly demonstrated using high-level multireference methods. By considering $60 \mathrm{BN}$ tetracene molecules with different doping configurations, we have first investigated the effects of varying the BN positions on the excitation energies relevant for SF, i.e., the first excited singlet, $S_{1}$, and the two low-lying triplet states, $T_{1}$ and $T_{2}$. It was found that, at least, $15 \mathrm{BN}$-doped structures fulfill the energetic conditions required to enhance the ability of a system to undergo SF. Within these potential SF candidates, the four BN-tetracene molecules 4,10, 2,3, 4,13 and 18,3 are especially attractive, since they exhibit exoergic SF (within the vertical excitation picture) in a descending order. The other eleven structures are also capable to undergo SF but with small endoergicity, that is, an additional thermal energy $\left(2 \mathrm{E}_{T 1}-\mathrm{E}_{S 1} \leq 0.3 \mathrm{eV}\right)$ must be provided to activate the SF process. Overall, to achieve the optimal energetic features for SF, the BN-dopants should be preferably introduced at the same ring or at both external rings of tetracene. In particular, the substitutional site $\mathrm{C}_{4}$ of tetracene appears to be the most indicated for chemical doping, since eight of the promising $\mathrm{SF}$ candidates has this position occupied by a B or $\mathrm{N}$ atom. A further important conclusion that can be drawn from our study is 
that the energy matching criteria for SF can be achieved even in the case of chromophores with reduced diradical character, which opens the opportunity to design SF molecules with low chemical reactivity.

By going beyond the energetic aspects of SF, we examine in detail the excitonic properties of the BN-tetracene chromophores to identify additional characteristics of the excited states that could be beneficial for solar cell applications. The computed binding energies of the singlet and triplet excitons show a strong dependence with BN position where binding energies tend to increase upon substitution. However, the 4,13 and 12,4 BN-tetracenes appear to be especially promising, since these systems exhibit rather weak electron-hole interactions in their singlet and triplet states when compared to the pristine molecule and other BN-doped structures, which is expected to facilitate the exciton dissociation into free charge carriers. Regarding this quantity, the 5,18, 4,13 and 12,4 BN-tetracenes appears to be especially promising, since these systems exhibit rather weak electron-hole interaction as compared to the pristine molecule and other BN-doped structures, which is expected to facilitate the exciton dissociation into free charge carriers. The noticeable variation of the exciton binding energy with respect to the $\mathrm{BN}$ positions is also reflected in the spatial distribution of the electron and hole. In fact, a strong modulation of the exciton size was observed for the whole series of BN-tetracenes, where delocalized excitons are more likely occur for the doped systems with large B,N separation. The analysis of the attachment/detachment densities also revealed that during the excitation process boron can either act as a donor or as an acceptor by transferring charge to or from the $\pi$ network upon the $\mathrm{S}_{0} \rightarrow \mathrm{S}_{1}$ electronic transition.

In conclusion, our results provide not only useful guidelines to design new efficient SF sensitizers for solar cell applications, but also provide a detailed understanding on how the BN-doping can be used to fine tune the excitonic properties and chemical stability, associated with the diradicaloid character, of tetracene. Taking the energetic and excitonic properties into consideration, we point out that, within the pool of $60 \mathrm{BN}$-tetracene systems investigated, compounds 2,3, 5,18 and 18,2 are likely the best SF sensitizers, as they exhibit the most suitable features: low exoergic or nearly isoergic relationship between singlet and the two triplets, triplet excitons with ideal energy $\left(\mathrm{E}_{T 1} \simeq 1.0-1.3\right.$ $\mathrm{eV}$ ) to share with conventional charge-absorbing layers of solar cells, reasonably small exciton binding energies, and good chemical stability. It is worth noting that the 2,3 and 5,18 BN-tetracenes share a common characteristic, that is, the $\mathrm{B}$ and $\mathrm{N}$ dopants are directly bound forming a B-N bond. These type of systems, also known as azaborines has been extensively studied and characterized experimentally with notable advances in synthesis techniques 8788 . More generally, our study proposes a systematic set of design principles based on computational estimates of relevant excitonic properties that might be easily extended to a broad range of molecular materials aiming to identify new singlet fission sensitizers. Thus, in light of the importance of the theoretical results presented herein for the development of organic solar cell technologies, we are confident that this study will motivate chemists to synthesize new acene-based azaborine molecules and related compounds, thereby expanding the highly limited collection of singlet fission chromophores.

Data availability. Additional data that support the findings of this study are available from the corresponding authors on reasonable request.

\section{Conflicts of interest}

There are no conflicts to declare.

\section{Acknowledgements}

The financial assistance of the Brazilian agencies Conselho Nacional de Desenvolvimento Científico e Tecnológico (CNPq) under Projects Nos. 307136/2019-1, 404337/2016-3, Fundação de Amparo à Pesquisa do Estado de São Paulo (FAPESP) under Projects Process No. 2019/25105-6 are gratefully acknowledged. The postdoctoral researches expresses their gratitude to Coordenação de Aperfeiçoamento de Pessoal de Nível Superior (CAPES) under Project No. 88882.161963/2014-01 by the fellowship. Authors are thankful to Vienna Scientific Cluster (VSC) under project 70376 for providing the high performance computational infrastructure and to FAPESP/Tianjin SPRINT program (project no. 2017/50157-4) for travel support. We are grateful also for computer time supplied by the School of Pharmaceutical Science and Technology, Tianjin University, China, on the computer cluster Arran.

\section{Notes and references}

1 M. B. Smith and J. Michl, Chem. Rev., 2010, 110, 6891-6936.

2 M. B. Smith and J. Michl, Annu. Rev. Phys. Chem., 2013, 64, 361-386.

3 B. J. Walker, A. J. Musser, D. Beljonne and R. H. Friend, Nat. Chem., 2013, 5, 1019-1024.

4 J. Lee, P. Jadhav, P. D. Reusswig, S. R. Yost, N. J. Thompson, D. N. Congreve, E. Hontz, T. Van Voorhis and M. A. Baldo, Acc. Chem. Res., 2013, 46, 1300-1311.

5 W. Shockley and H. J. Queisser, J. Appl. Phys., 1961, 32, 510519.

6 M. C. Hanna and A. J. Nozik, J. Appl. Phys., 2006, 100, 074510.

7 J. Lee, P. Jadhav and M. A. Baldo, Appl. Phys. Lett., 2009, 95, 033301.

8 A. Rao, M. W. B. Wilson, J. M. Hodgkiss, S. Albert-Seifried, H. BÃd'ssler and R. H. Friend, J. Am. Chem. Soc. , 2010, 132, 12698-12703.

9 O. E. Semonin, J. M. Luther, S. Choi, H.-Y. Chen, J. Gao, A. J. Nozik and M. C. Beard, Science, 2011, 334, 1530-1533.

10 P. J. Jadhav, A. Mohanty, J. Sussman, J. Lee and M. A. Baldo, Nano Lett., 2011, 11, 1495-1498.

11 D. N. Congreve, J. Lee, N. J. Thompson, E. Hontz, S. R. Yost, P. D. Reusswig, M. E. Bahlke, S. Reineke, T. Van Voorhis and M. A. Baldo, Science, 2013, 340, 334-337.

12 T. C. Wu, N. J. Thompson, D. N. Congreve, E. Hontz, S. R. Yost, T. Van Voorhis and M. A. Baldo, Appl. Phys. Lett., 2014, 104, 193901. 
13 B. Ehrler, M. W. B. Wilson, A. Rao, R. H. Friend and N. C. Greenham, Nano Lett., 2012, 12, 1053-1057.

14 S. Singh, W. J. Jones, W. Siebrand, B. P. Stoicheff and W. G. Schneider, J. Chem. Phys., 1965, 42, 330-342.

15 M. W. B. Wilson, A. Rao, B. Ehrler and R. H. Friend, Acc. Chem. Res., 2013, 46, 1330-1338.

16 E. Busby, T. C. Berkelbach, B. Kumar, A. Chernikov, Y. Zhong, H. Hlaing, X.-Y. Zhu, T. F. Heinz, M. S. Hybertsen, M. Y. Sfeir, D. R. Reichman, C. Nuckolls and O. Yaffe, J. Am. Chem. Soc. , 2014, 136, 10654-10660.

17 N. R. Monahan, D. Sun, H. Tamura, K. W. Williams, B. Xu, Y. Zhong, B. Kumar, C. Nuckolls, A. R. Harutyunyan, G. Chen, H.-L. Dai, D. Beljonne, Y. Rao and X.-Y. Zhu, Nat. Chem., 2017, 9, 341-346.

18 S. T. Roberts, R. E. McAnally, J. N. Mastron, D. H. Webber, M. T. Whited, R. L. Brutchey, M. E. Thompson and S. E. Bradforth, J. Am. Chem. Soc. , 2012, 134, 6388-6400.

19 R. D. Pensack, A. J. Tilley, S. R. Parkin, T. S. Lee, M. M. Payne, D. Gao, A. A. Jahnke, D. G. Oblinsky, P.-F. Li, J. E. Anthony, D. S. Seferos and G. D. Scholes, J. Am. Chem. Soc. , 2015, 137, 6790-6803.

20 G. B. Piland and C. J. Bardeen, J. Chem. Phys. Lett., 2015, 6, 1841-1846.

21 C. Jundt, G. Klein, B. Sipp, J. Le Moigne, M. Joucla and A. Villaeys, Chem. Phys. Lett., 1995, 241, 84-88.

22 S. W. Eaton, L. E. Shoer, S. D. Karlen, S. M. Dyar, E. A. Margulies, B. S. Veldkamp, C. Ramanan, D. A. Hartzler, S. Savikhin, T. J. Marks and M. R. Wasielewski, J. Am. Chem. Soc. , 2013, 135, 14701-14712.

23 A. K. Le, J. A. Bender, D. H. Arias, D. E. Cotton, J. C. Johnson and S. T. Roberts, J. Am. Chem. Soc. , 2018, 140, 814-826.

24 J. C. Johnson, A. J. Nozik and J. Michl, J. Am. Chem. Soc. , 2010, 132, 16302-16303.

25 A. J. Musser, M. Maiuri, D. Brida, G. Cerullo, R. H. Friend and J. Clark, J. Am. Chem. Soc. , 2015, 137, 5130-5139.

26 O. Varnavski, N. Abeyasinghe, J. Aragó, J. J. Serrano-Pérez, E. Ortí, J. T. López Navarrete, K. Takimiya, D. Casanova, J. Casado and T. Goodson, J. Phys. Chem. Lett., 2015, 6, 1375-1384.

27 S. Lukman, J. M. Richter, L. Yang, P. Hu, J. Wu, N. C. Greenham and A. J. Musser, J. Am. Chem. Soc. , 2017, 139, 1837618385.

28 I. Paci, J. C. Johnson, X. Chen, G. Rana, D. Popović, D. E. David, A. J. Nozik, M. A. Ratner and J. Michl, J. Am. Chem. Soc. , 2006, 128, 16546-16553.

29 T. Minami and M. Nakano, J. Chem. Phys. Lett., 2012, 3, 145150.

30 K. J. Fallon, P. Budden, E. Salvadori, A. M. Ganose, C. N. Savory, L. Eyre, S. Dowland, Q. Ai, S. Goodlett, C. Risko, D. O. Scanlon, C. W. M. Kay, A. Rao, R. H. Friend, A. J. Musser and H. Bronstein, J. Am. Chem. Soc., 2019, 141, 13867-13876.

31 J. L. Ryerson, A. Zaykov, L. E. Aguilar Suarez, R. W. A. Havenith, B. R. Stepp, P. I. Dron, J. Kaleta, A. Akdag, S. J. Teat, T. F. Magnera, J. R. Miller, Z. Havlas, R. Broer, S. Faraji,
J. Michl and J. C. Johnson, J. Chem. Phys., 2019, 151, 184903.

32 M. Nakano, The Chemical Record, 2017, 17, 27-62.

33 K. Bhattacharyya and A. Datta, J. Phys. Chem. C, 2019, 123, 19257-19268.

34 S. Ito, T. Nagami and M. Nakano, J. Phys. Chem. Lett., 2016, 7, 3925-3930.

35 Y. Chen, L. Shen and X. Li, J. Phys. Chem. A, 2014, 118, 57005708.

36 K. Bhattacharyya, S. M. Pratik and A. Datta, J. Phys. Chem. C, 2015, 119, 25696-25702.

37 S. Ito, T. Minami and M. Nakano, J. Phys. Chem. C, 2012, 116, 19729-19736.

38 T. Zeng, N. Ananth and R. Hoffmann, J. Am. Chem. Soc. , 2014, 136, 12638-12647.

39 M. Pinheiro, L. F. A. Ferrao, F. Bettanin, A. J. A. Aquino, F. B. C. Machado and H. Lischka, Phys. Chem. Chem. Phys., 2017, 19, 19225-19233.

40 S. A. Mewes, J.-M. Mewes, A. Dreuw and F. Plasser, Phys. Chem. Chem. Phys., 2016, 18, 2548-2563.

41 S. A. Bäppler, F. Plasser, M. Wormit and A. Dreuw, Phys. Rev. A, 2014, 90, 052521.

42 F. Plasser, M. Wormit and A. Dreuw, J. Chem. Phys., 2014, 141, 024106.

43 F. Plasser and H. Lischka, J. Chem. Theory Comput. , 2012, 8, 2777-2789.

44 F. Plasser and A. Dreuw, J. Phys. Chem. A, 2015, 119, 10231036.

45 F. Plasser, S. A. Mewes, A. Dreuw and L. González, J. Chem. Theory Comput. , 2017, 13, 5343-5353.

46 S. Chakraborty, P. Kayastha and R. Ramakrishnan, J. Chem. Phys., 2019, 150, 114106.

47 C. Møller and M. S. Plesset, Phys. Rev., 1934, 46, 618-622.

48 F. Weigend and M. Häser, Theor. Chem. Acc., 1997, 97, 331340.

49 F. Weigend and R. Ahlrichs, Phys. Chem. Chem. Phys., 2005, 7, 3297-3305.

50 K. Ruedenberg, L. M. Cheung and S. T. Elbert, Int. J. Quantum Chem., 1979, 16, 1069-1101.

51 C. Angeli, R. Cimiraglia and J.-P. Malrieu, J. Chem. Phys., 2002, 117, 9138-9153.

52 P. G. Szalay, T. Müller, G. Gidofalvi, H. Lischka and R. Shepard, Chem. Rev., 2012, 112, 108-181.

53 A. Bunge, J. Chem. Phys. , 1970, 53, 20-28.

54 E. R. Davidson, The World of Quantum Chemistry, Dordrecht, 1974, pp. 17-30.

55 S. R. Langhoff and E. R. Davidson, Int. J. Quantum Chem., 1974, 8, 61-72.

56 F. Neese, WIREs Comput Mol Sci, 2012, 2, 73-78.

57 H. Lischka, T. Müller, P. G. Szalay, I. Shavitt, R. M. Pitzer and R. Shepard, WIREs Comput Mol Sci, 2011, 1, 191-199.

58 P. G. Szalay, T. Muller and H. Lischka, Phys. Chem. Chem. Phys., 2000, 2, 2067-2073.

59 Columbus, an Ab Initio Electronic Structure Program, Release 
7.0, 2017, https: / / www. univie.ac.at/columbus/.

60 H. Dachsel, H. Lischka, R. Shepard, J. Nieplocha and R. J. Harrison, J. Comput. Chem. , 1997, 18, 430-448.

61 F. Plasser, J. Chem. Phys., 2020, 152, 084108.

62 F. Plasser, B. Thomitzni, S. A. Bäppler, J. Wenzel, D. R. Rehn, M. Wormit and A. Dreuw, J. Comput. Chem., 2015, 36, 16091620.

63 M. Head-Gordon, Chem. Phys. Lett., 2003, 372, 508-511.

64 A. V. Luzanov and O. V. Prezhdo, Int. J. Quantum Chem., 2005, 102, 582-601.

65 M. Head-Gordon, A. M. Grana, D. Maurice and C. A. White, J. Phys. Chem., 1995, 99, 14261-14270.

66 F. Plasser, S. A. BÃd'ppler, M. Wormit and A. Dreuw, J. Chem. Phys., 2014, 141, 024107.

67 P. Kimber and F. Plasser, Phys. Chem. Chem. Phys., 2020, 22, 6058-6080.

68 E. Clar, The Aromatic Sextet, J. Wiley, 1972.

69 A. Das, T. Müller, F. Plasser and H. Lischka, J. Phys. Chem. A, 2016, 120, 1625-1636.

70 Y. Tomkiewicz, R. P. Groff and P. Avakian, J. Chem. Phys., 1971, 54, 4504-4507.

71 D. Biermann and W. Schmidt, J. Am. Chem. Soc. , 1980, 102, 3163-3173.

72 B. Hajgato, M. Huzak and M. S. Deleuze, J. Phys. Chem. A, 2011, 115, 9282-9293.

73 J. S. A. Ishibashi, A. Dargelos, C. Darrigan, A. Chrostowska and S.-Y. Liu, Organometallics, 2017, 36, 2494-2497.
74 M. J. S. Dewar and W. H. Poesche, J. Am. Chem. Soc. , 1963, 85, 2253-2256.

75 B. O. Roos, K. Andersson and M. P. Fülscher, Chem. Phys. Lett., 1992, 192, 5-13.

76 M. Boggio-Pasqua, M. J. Bearpark, M. Klene and M. A. Robb, J. Chem. Phys., 2004, 120, 7849-7860.

77 K. C. Krishnapriya, A. J. Musser and S. Patil, ACS Energy Lett., 2019, 4, 192-202.

78 W. Schmidt, J. Chem. Phys., 1977, 66, 828-845.

79 L. Crocker, T. Wang and P. Kebarle, J. Am. Chem. Soc. , 1993, 115, 7818-7822.

80 J. Xia, S. N. Sanders, W. Cheng, J. Z. Low, J. Liu, L. M. Campos and T. Sun, Adv. Mater., 2017, 29, 1601652.

81 L. Zhu, Y. Yi and Z. Wei, J. Phys. Chem. C, 2018, 122, 2230922316.

82 J.-L. Bredas, Mater. Horiz, 2014, 1, 17-19.

83 I. Hill, A. Kahn, Z. Soos and J. R.A. Pascal, Chem. Phys. Lett., 2000, 327, 181 - 188.

84 P. Kimber and F. Plasser, Phys. Chem. Chem. Phys., 2020, 22, 6058-6080.

85 T. M. Clarke and J. R. Durrant, Chem. Rev., 2010, 110, 67366767.

86 T. Etienne, X. Assfeld and A. Monari, J. Chem. Theory Comput. , 2014, 10, 3896-3905.

87 P. G. Campbell, A. J. V. Marwitz and S.-Y. Liu, Angew. Chem. Int. Ed., 2012, 51, 6074-6092.

88 Z. X. Giustra and S.-Y. Liu, J. Am. Chem. Soc. , 2018, 140, 1184-1194. 


\section{Graphical abstract description:}

A fine tunning of the energy levels relevant for singlet fission is attainable by iew Article Online $_{\text {. }}$ doping tetracene with a nitrogen-boron pair. DOI: 10.1039/C9TC06581D 


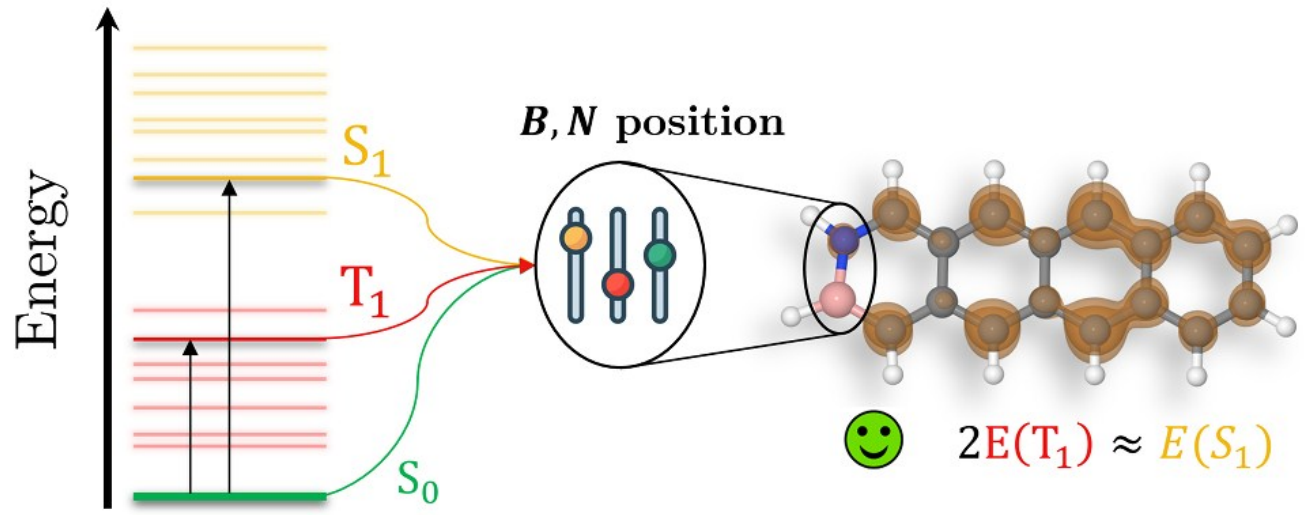

Ground State 